\title{
ESBOZO HISTÓRICO ACERCA DE LA PREGUNTA POR LA VERDAD
}

\author{
Alejandro Rojas Jiménez
}

Como breve estudio preliminar que busca servir de preludio a unas jornadas que analizarán la cuestión por la verdad en los grandes textos de la historia de la filosofía, quizás sea pertinente un esbozo histórico introductorio que prepare a los oyentes no ya tanto a una sucesión de respuestas, sino al diálogo teórico por entender el sentido de lo verdadero.

\section{Palabras claves:}

Verdad, Ser, Enuntiatio, imaginación, razón

As a brief preliminary study intended to serve as a prelude to a conference to discuss the issue of truth in the great texts of history of philosophy, it may be relevant an introductory historical sketch which will prepare listeners not for a succession of responses but the theoretical dialogue to understand the meaning of truth.

\section{Keywords:}

Truth, Being, Enuntiatio, Imagination, reason

\section{Índice de contenidos:}

1. ¿Qué es la verdad? Primer paso: la enuntiatio.

2. Segunda paso. La verdad como mera aísthesis.

3. Tercer paso. Del recuerdo a la desocultación, y de la desocultación a la adecuación.

4. Cuarto paso. La búsqueda de claridad lógica: del criterio de verificabilidad y el criterio de sentido.

5. Quinto paso. La imaginación.

6. Sexto paso. El papel fundante de la imaginación

7. Séptimo paso. El paso hacia atrás hacia el ámbito presencial

*Ludwig-Maximilians-Universität München. rojasj_a@yahoo.es 


\section{1. ¿Qué es la verdad? Primer paso: la enuntiatio}

La verdad, ¿qué es? debemos empezar este simposio mostrando, ya de entrada, que la pregunta por la verdad no busca ningún sujeto, que la verdad no se predica de los sujetos. De los sujetos podemos decir muchas cosas. Decimos en primer lugar que son (hypokeimenon), pero también decimos por ejemplo que tal sujeto es alto, y en este sentido señalamos una cualidad del sujeto. Cualidades, cantidades, relaciones ${ }^{1}$... son muchos los sentidos en los que un predicado se dice de un sujeto: tal sujeto es hijo de, tal sujeto es gordo... Pero ninguno de estos sentidos es la verdad. De un sujeto no podemos decir que es verdad. La verdad se refiere a la enunciación.

Quizás ciertamente se pueda decir cuando verdad tiene el sentido opuesto a mentira, como cuando juramos que nuestro amor es verdadero. Pero no es este sentido secundario de la verdad por el que nos interrogamos, sino por la verdad del entendimiento, esto es, la verdad que se opone, no a la mentira, sino a lo no entendido. Ni siquiera a lo mal entendido, sino a lo no compareciente en ningún sentido al entendimiento. Esto entendido podrá ser a su vez verdadero o falso, pues verdad tiene dos acepciones: en un sentido reducido es lo bien entendido, y en un sentido amplio lo entendido a secas.

Esta es la primera indicación clásica sobre la cuestión que nos ocupa: que lo verdadero no se dice de las $\operatorname{cosas}^{2}$, de los sujetos, sino de los sujetos en relación con nuestro entendimiento. La verdad tiene que ver con entender los sujetos. No tiene que ver con los sujetos, sino con lo que decimos de ellos.

Tomando el camino inverso a la Metafísica $^{3}$, no debemos dirigir nuestra atención hacia el sujeto de los predicados. Tampoco al predicado; sino a la enunciación, esto es, a la unión de un predicado a un sujeto según la fórmula «S es $\mathrm{P} »$. Debemos retener, y es nuestro primer paso en la tarea de dilucidar qué es la verdad, que lo verdadero se predica

\footnotetext{
${ }^{1} \mathrm{Si}$ antes habíamos hablado de la categoría de cualidad, ahora estamos utilizando la categoría de lugar. Aristóteles recoge las siguientes categorías: cantidad, cualidad, relación, lugar, tiempo posición, posesión, acción y pasión. A esta lista habría que añadir la de sustancia, el primer sentido del ser.

${ }^{2}$ Cfr, Metafísica, VI, 4, 1027 b -1028a

${ }^{3}$ Cfr, Metafísica, VI, 4, 1027 b -1028a
} 
de la enunciación «tal sujeto $(\mathrm{S})$ es tal predicado $(\mathrm{P}){ }^{4}{ }^{4}$. El reino de lo verdadero y lo falso es el reino de la combinación, de la síntesis.

Mención a parte merecen las entidades simples. Éstas o se captan o no se captan, pero no cabe la falsedad. Lo inmediato es siempre verdadero, pero es una verdad trivial: "acerca de las cosas que son puro ser y actos no es posible engañarse, sino que se piensa en ellas o no"s. No hay falsedad en ver algo alto, o en que sepa algo agrio (¡ni siquiera es falso que sepa agrio un dulce!); cosa distinta es si lo que enunciamos es «este dulce es agrio o este árbol es alto».

Pongamos el ejemplo siguiente: se cuela un pájaro en el aula donde tiene lugar un Simposio sobre Teorías de la Verdad, y un participante avisa que hay un gato en el aula. No habría falsedad en lo que ha visto, sino en lo que dice que ha visto. Decimos que es falso porque al mirar nosotros vemos un pájaro en lugar de un gato, pero la falsedad no se mide por lo que él ha visto: siendo verdad que él ha visto un gato, es falso que lo haya, porque cuando miramos hacia donde señala el participante vemos un pájaro. Lo que hay es un no-captar un gato (más que falsedad hay accidente, que diría Aristóteles). Quizás sea más claro este otro ejemplo: «veo el palo doblado al sumergirlo en el río». Si nos preguntamos por la verdad o falsedad no tendremos más remedio que aceptar que si ve un palo doblado en el río, entonces ve un palo doblado en el río. Sin embargo el enunciado tal palo (S) está doblado (P) sería falso. Dicho sin ejemplos: en lo inmediato no hay falsedad. Por eso será para los racionalistas y empiristas tan importante precisar lo que significa una idea simple.

\section{Segundo paso. La verdad no es mera aísthesis}

El hecho de que la verdad se diga de lo que se dice sobre lo que se capta sitúa la experiencia en un punto originario. Siguiendo el ejemplo anterior, decíamos que es falso porque al mirar nosotros veíamos un pájaro. Tanto es así, que en más de una ocasión la filosofía ha recordado que la experiencia es el punto de partida del conocimiento. Pero que lo verdadero y lo falso no se diga del ente en cuanto ente, sino de lo presenciado (acto) y lo enunciado (combinación) ${ }^{6}$, no debería hacer

\footnotetext{
${ }^{4}$ Cfr, Metafísica, IX, 10, 1051 b - 1052 a

${ }^{5}$ Metafísica, IX, 10, 1051b-1052a

${ }^{6}$ Metafísica, VI cap 4
} 
olvidar que se guarda cierta correspondencia entre lo no-presente (hypokeimenon) y lo que se capta, que lo primero guarda con respecto a lo segundo cierto poder causal al que corresponde lo presenciado: el sujeto (hypokeimenon) permanece oculto, pero sustentado lo que se predica de él. La pregunta por la verdad nos lleva más allá de la aísthesis ${ }^{7}$ en busca del fundamento de la correspondencia. Si uno dice que vio un gato, y nosotros vemos un pájaro ¿se trata entonces de un problema de percepción? No, en el enunciado hay un pájaro en el aula la clave no es la percepción, sino la combinación: predicamos algo de un sujeto real, unimos un predicado a un sujeto. El asunto es. ¿Hemos hecho bien esto? La validez de la unión no puede percibirse, y tiene un fundamento externo.

Para Platón, de hecho, la percepción es tan irrelevante como que, según él, el entendimiento lo único que hace es recordar ${ }^{8}$, como muestra Sócrates en su diálogo con el esclavo, algo que nadie le ha enseñado ${ }^{9}$ ni que ha sido aprendido, sino algo previo a la experiencia, algo que se encuentra mantenido en la memoria colectiva que guarda el lógos ${ }^{10}$. Ciertamente no vale para el ejemplo anterior, sino para rastrear el sentido de nociones como justicia o libertad. Siendo así la verdad y la falsedad en Platón tienen que ver con ese dominio preexistente que Emilio Lledó nos enseñó a ver como la lengua que hablamos. Tal sería la razón de que la única condición que puso Sócrates para demostrar su mayéutica fuera la de que el esclavo hablara griego.

Aristóteles parte de este esquema platónico, si bien es cierto que ha observado que, para salvar la pregunta por la verdad de un relativismo que afirme que lo verdadero es lo que se siente, no se precisa crear un topos noetos, basta sencillamente con hacer ver que la pregunta por la verdad no se dirige hacia la cosa sentida $y$, en general, percibida (que siempre es verdadera), sino hacia el enunciado.

\footnotetext{
${ }^{7}$ Como explica Platón en el Teeteto al comentar la sentencia de Protágoras, la aísthesis es la visión, la audición, el olfato, el placer, el dolor, el deseo, el amor...

${ }^{8}$ Fedón $72 \mathrm{e}-77 \mathrm{a}$

${ }^{9}$ Menón $81 \mathrm{c}-86 \mathrm{~b}$

10 "Es en el lenguaje donde se ha preexistido (...) Hablar es haber ido dejando reposar toda la preexistente sabiduría de la palabra, haberse ido enriqueciendo con la única puerta consciente que nos ha dejado abierta el pasado y, de pronto, utilizar de nuevo ese legado para sintetizar, en un «acto de habla», la preexistencia que hace posible, y la existencia que realiza"; Emilio Lledó, La memoria del Lógos, Taurus, 1996, Madrid
} 


\section{Tercer paso. Del recuerdo a la desocultaicón, y de la desocultación a la adecuación}

No es mera aísthesis porque hay cierto poder causal en la realidad que debemos destacar. De tal modo que la verdad nombraría una correspondencia entre lo pensado y la realidad que fundamenta lo pensado. A menudo se malinterpreta este sentido de la correspondencia. Correspondencia no nombra una identidad donde la idea y la realidad se vuelven indiferentes uno para el otro, la correspondencia debe ser pensada desde la mayoría de edad dialéctica, como una relación de identidad donde se mantiene la diferencia y la oposición. No es por otro lado nada difícil si no pensamos la identidad como mera generalización, quiero decir, cuando digo que en esta sala hay 50 oyentes estoy efectivamente prescindiendo de las diferencias, pero existen otros modos de unificar menos agresivos contra las diferencias. Schelling en las lecciones privadas de Stuttgart pone, como ejemplo de cómo debe ser pensada la relación de identidad, la unidad del organismo: decimos que el estómago y el cerebro son diferentes, pero constituyen junto con el resto de órganos y sus funciones una unidad. Del mismo modo el sujetoque-piensa y la realidad-pensada son diferentes, pero constituyen una unidad: la unidad de lo pensado. En la unidad de lo pensado conviven: la idea y la realidad como diferentes.

Se trata de resaltar, utilizaré otra expresión muy conocida en este ligero repaso histórico que sólo busca servir como introducción a las jornadas que nos ocupan, de advertir una adecuación, una adaequatio: el entendimiento se adecúa a las cosas.

Si bien, es cierto que la adecuación introduce algo que no estaba en Aristóteles, al menos según la formulación inicial de su autor, al dar entrada a una nueva idea de Dios que habría creado el mundo ex nihilo. Tomás de Aquino, le damos la vuelta a la carta, establece una correspondencia entre el entendimiento y una realidad que ya responde a su vez a una relación anterior con el entendimiento divino ${ }^{11}$, esto es, una

\footnotetext{
11 "una cosa natural, puesta entre los dos entendimientos, se dice verdadera por la adecuación a ambos; pues, por la adecuación al entendimiento divino se dice verdadera en cuanto realiza aquello para lo que está ordenada por el entendimiento divino, como es puesto de manifiesto por Anselmo en el libro De veritate y por Agustín en el libro De vera religione y por Avicena en la definición aducida, a saber: la verdad de cada cosa es la propiedad de su ser que le ha sido establecido. En cambio, por su adecuación al entendimiento humano, una cosa se dice verdadera en cuanto está naturalmente constituida para formar una estimación verdadera de sí misma; como, por el contrario,
} 
realidad que es fundamento fundado por un entendimiento superior, de modo que, cito a Tomás, "todo ente, en efecto, está adecuado al entendimiento divino, y pudiendo adecuar a sí el entendimiento humano, y a la inversa" $"$.

Lo interesante de esta concepción es que es dependiente de un hallazgo especulativo de una enrome relevancia para la tradición filosófica: el plano del acto existencial más allá del acto sustancial. Tomás introduce el esse. El plano del ente es el plano de la cosa, de la sustancia, al que Tomás añade el plano de la existencia: el ente existe. Existe de tal manera que puede ser conocido por el hombre, este ser conocido sería el fin de la existencia, la verdad ${ }^{13}$, pero un fin posterior a su existencia, la cual es independiente del entendimiento humano. La realidad sería así "adecuabilidad", la verdad adecuación, y el entendimiento adecuable.

O dicho con otras palabras más modernas: la representación es el ansia de la realidad ${ }^{14}$. Observen que es mucho más radical Tomás que Schopenhauer, la realidad sólo existe como ansia por dejarse representar. Eso es lo que ha creado el Dios tomista: un anhelo. Pero como lo ha creado Dios, ese ansia es independiente de nosotros, es la esencia de una realidad que existe por sí, que no nos debe su existencia ¡aquí está ya aquella dualidad que impulsará toda la filosofía moderna! Ha sido Tomás, aunque posiblemente sin que sea su intención, quien ha introducido eso que Schelling denominará en sus lecciones de Stuttgart como "aquella fuerza oscura que permanece como fondo de toda existencia, lo in-exterminable que ningún disolvente disuelve" ${ }^{15}$ y que no puede ser pensada sin su relación al entendimiento y la luz.

se dicen falsas las cosas que están naturalmente constituidas para parecer lo que no son o como no son, según se dice en el libro 5 de la Metafísica" De veritate, q2, c

${ }^{12}$ De veritate $\mathrm{q} 2$, ad1

13 "oporter igitur ultimum finem universi esse bonum intellectus, hoc autem est veritas" Contra gentes I, 1

14 "El fin del universo es ser conocido por el hombre", Juan A García, "Prólogo", en L. Polo, El orden predicamental, Cuadernos de anuario filosófico, Pamplona, 2005, p 7

${ }^{15} \mathrm{SW} \mathrm{I} / 7441$ 
4. Cuarto paso. La búsqueda de claridad lógica. Del criterio de verificabilidad y el criterio de sentido

¿Pero acaso debe llevar la pregunta por la verdad a hablar de oscuridad y tinieblas? ¿No debería ser precisamente esta cuestión la que albergara mayor claridad? ¿Qué lugar merece tener lo oscuro allí donde sólo debería haber luminosidad? ¿Acaso no cabría examinar la cuestión por la verdad de un modo que renunciase de entrada a oscuridades? Existió desde luego un intento semejante, un empirismo lógico que subordinó el criterio de verificabilidad al criterio de sentido como recurso para eludir oscuridades y entelequias. La pregunta que se imponía resolver, pues la cosa va de enunciados, era qué tipo de enunciados deberíamos aceptar.

Hay muchos tipos de enunciados. Hay enunciados en los que claramente la verdad no se decide por la experiencia. De hecho son los más claros, aquellos donde la falsedad es imposible: en enunciados del tipo "todo los cuerpos son extensos" 16 no cabe falsedad porque lo que predican se encuentra ya incluido en el sujeto. Este tipo de enunciados que expresan una verdad de razón (Leibniz) o relación de ideas (Hume) no pueden ser falsos, ya que siempre expresan una verdad necesaria, compartiendo entre sí el que para afirmar su verdad no es preciso recurrir a la experiencia ya que su contradictorio es impensable. El problema de estos enunciados analíticos es que no se pueden aplicar a las cuestiones de hechos.

¿Qué ocurre con aquellos enunciados que expresan un juicio sintético y que, sin embargo, no pueden ser verificados mediante la experiencia? Un verificacionista no tendría ningún problema en decir sencillamente que un enunciado que no puede ser verificado empíricamente carece de sentido; aunque ya, el que posiblemente sea el más grande de entre los analíticos, había corregido a todos los positivistas lógicos que agrupaban el Círculo de Viena afirmando que aunque son sinsentidos, unsinnig, no carecen de sentido, sinnlos ${ }^{17}$.

Es desmesurado identificar el criterio de verificabilidad con el criterio de sentido haciendo de dos cuestiones una sola. Una proposición del estilo: nihil est sine ratione para ellos carece de sentido (absurda) simplemente porque no pueden decidir empíricamente su veracidad. Para ellos «nihil est sine ratione» vale tanto como la

\footnotetext{
${ }^{16}$ KRV IV, B 11

${ }^{17}$ Tractatus, 4.461-4.4611
} 
proposición «la mesa está sobre la raíz de dos». O, por poner otro ejemplo, la proposición «sólo tiene sentido la proposición verificable empíricamente» vale tanto como «la mesa está sobre la raíz de dos». $\mathrm{Si}$ bien no sólo tienen que enfrentarse al problema de que se revuelva contra sí su propia tesis, sino que también deben hacer frente a otras dificultades. Ya que ¿qué significa que puede ser verificada empíricamente?

Si tiene un sentido práctico, entonces resulta, que la proposición «hay montañas en la cara oculta de la luna» no es verdadera (sino absurda) cuando no se tienen los medios suficientes para comprobarlo (era absurda y sin sentido así esta afirmación en el siglo VII después de Cristo). Algo que ni siquiera ellos estuvieron dispuestos a admitir y por lo que se adoptó el criterio de la verificabilidad "en principio": una proposición es verificable si sabemos qué observaciones podrían decidir su verdad, aunque no dispongamos de medios para hacerlo.

Pero el criterio verificacionista también encuentra dificultades a la hora de analizar el alcance de su propuesta, ya que, por ejemplo, las proposiciones universales (y con ello las leyes científicas o proposiciones tales como «los hombres son mortales) son imposibles de verificar. Lo que ha conducido a reducir el alcance: una proposición tiene sentido si es posible que la experiencia la haga probable. La probabilidad sustituye finalmente a la verificabilidad. Pero de este modo el neopositivismo no supone ningún avance con respecto al empirismo tal y como lo presentó Hume.

Una solución frente al criterio verificacionista podría ser el falsacionismo de Popper: un enunciado tiene sentido sólo si puede ser falsado por la experiencia y, de hecho, no ha sido falsado. No se trata pues de apuntar hacia la confirmación del enunciado, como diría Carnap, sino hacia su refutación. Claro que si esto significa que la ciencia no puede confirmar, sino sólo falsar (Ayer, Kuhn, Nagel) Lakatos creerá necesario distinguir entre un falsacionismo dogmático y otro metodológico que admita cierta verificabilidad de las hipótesis ${ }^{18}$.

El falsacionismo permite que enunciados como «todos los hombres son mortales» o leyes universales como «la fuerza que ejerce

\footnotetext{
${ }^{18}$ dentro de éste distinguirá a su vez entre una versión ingenua (Popper) y otra refinada (Lakatos) que no acepte que toda teoría experimentalmente falsable sea científica, sino que debería poseer además un exceso de contenido empírico corroborado con relación a la teoría predecesora
} 
una partícula puntual con masa $m_{1}$ sobre otra con masa $m_{2}$ es directamente proporcional al producto de las masas, e inversamente proporcional al cuadrado de la distancia que las separa» se salven como enunciados aceptables para la ciencia, cosa que no ocurría con el criterio verificacionista.

Debe notarse que la pregunta por la verdad, en estos momentos, ha dado un buen giro, pues tanto la verificación como la falsación son extrínsecas a la adecuación (ente-pensado) e intrínsecas a lo pensado. No sirven para entender porqué decimos de algo que es verdadero o falso, sino que procede más bien fijando las condiciones a las que debemos someternos antes de discutir la verdad o falsedad de un enunciado. El resultado es sin embargo insuficientemente abarcador: deja fuera enunciados del tipo «el mayor bien que puede existir en un Estado es el de tener verdaderos filósofos»». ¿Debemos prescindir sin más de este tipo de enunciados sencillamente porque no se ajusten a los criterios de verdad acordados? Quizás no valgan para la ciencia, pero no por ello dejan de ser enunciados con valor de verdad.

\section{Quinto paso. La imaginación}

El auténtico problema es que en este tipo de enunciados no recurrimos a la experiencia para decidir su verdad o falsedad, sino que en lugar de la experiencia, el protagonismo recae sobre la imaginación. Así en el ejemplo anterior se trata de imaginar un Estado con buenos filósofos y un Estado sin buenos filósofos y ver si éstos, efectivamente, deben ser considerados el mayor bien del Estado. Pero la imaginación quiere evitarse. En el momento en que hacemos entrar la imaginación y nos alejamos de los sentidos, pareciera que nos adentramos en un universo subjetivo que no puede conducir a ninguna respuesta definitiva. Sin embargo, la imaginación está más cerca de los enunciados "aceptables" de lo que pensamos. Así, por ejemplo, se dice que es verdadero el enunciado que afirma la redondez de la Tierra, aún cuando la redondez de ésta no se ve ni se huele. La imaginamos redonda. Pero sobre todo: la creencia en la existencia de un universo externo que no depende de nuestra percepción y que existiría aunque nosotros, y toda 
criatura sensible, estuviéramos ausentes o hubiéramos sido aniquilados ${ }^{19}$ no se funda en la percepción, sino en nuestra imaginación.

Lo mismo ocurre con la idea de Espíritu como sustancia del yo, no es sino la proyección de la imaginación en relación con la memoria. ¿Acaso son sencillamente ideas regulativas? Quizá nuestro conocimiento sea limitado para decidir científicamente si enunciados de este tipo son verdaderos o falsos, pero eso sólo dice que no podemos decidirlo científicamente, y no que no sean verdaderos o falsos, y mucho menos que sean carentes de sentido.

Es cierto que ciertos realismos aceptan sin más que el universo independiente es precisamente el que se ve. Para ellos los filósofos tradicionales gustan de hablar de sustancias como el salvaje habla de una gran tortuga o de un gran elefante que sostiene el mundo ${ }^{20}$. Para ellos la verdad es lo que es según la primera clasificación que recogía Santo Tomás de Aquino en su De veritate.

El realismo actual no tiene porqué ser ingenuo, no tiene porqué olvidar algo que sabemos desde Aristóteles: la distinción entre el ente en cuanto ente y el ente en cuanto que es conocido. En este sentido un realismo tan actual como el de Tarski "defiende que hay objetos que pueden ser denotados, sin compromiso ontológico con la existencia real de tales objetos" $"$. Su teoría de la verdad es tan compatible con el realismo ingenuo como con el realismo crítico o el idealismo epistemológico.

En filosofía no debe olvidarse algo tan básico como la distinción aristotélica entre el ente en tanto ente y el ente en tanto que conocido. Si no se hace, entonces lo propio no es el realismo ingenuo, sino afirmar con Hume que el conocimiento de la naturaleza se debe

\footnotetext{
19 "It seems also evident, that, when men follow this blind and powerful instinct of nature, they always suppose the very images, presented by the senses, to be the external objects, and never entertain any suspicion, that the one are nothing but representations of the other. This very table which we see white, and which we feel hard, is believed to exist, independent of our perception, and to be something external to our mind, which perceives it. Our presence bestows not being on it: our absence does not annihilate it. It preserves its existence uniform and entire, independent of the situation of intelligent beings, who perceive or contemplate it" An enquiry concerning human understanding, 12,1

${ }^{20}$ An essay concerning human understanding, Part 2, cap 23, n 2

21 Pascual Martínez Freire, "la teoría de la verdad de Alfred Tarski", Contrastes, volumen V, Málaga, 2000, p 110
} 
limitar a las impresiones que de ella tenemos, pero esto significa precisamente aceptar que no cabe auténtico conocimiento y que toda ciencia, en tanto que cada una de sus teorías va más allá de lo percibido, dice más de lo que debe decirse. De este modo, enunciados como "saldrá el Sol mañana», «el agua se evapora a 100 grados centígrados» o «la servilleta de papel de mi cocina absorbe el agua», van más allá de lo que debe decirse, ya que en todos estos casos estamos imaginando que la naturaleza se comporta de modo uniforme, y no esperamos que las leyes cambien repentinamente. Hume será tremendamente radical: sobre cuestiones de hecho no cabe auténtico conocimiento porque no cabe demostración. Sólo habría demostración, y por ende auténtico conocimiento en las matemáticas y la lógica (relaciones de ideas) ${ }^{22}$.

\section{El papel fundante de la imaginación}

A partir de Hume se abren dos vías: la que se cierra a la imaginación convirtiendo en su estandarte el conocido como "microscopio de Hume"23, y la línea filosófica que se abre a la imaginación y descubre su papel fundante. La primera llega hasta el atomismo lógico, la segunda es la línea de la imaginación trascendental kantiana.

Contra el atomismo lógico debe decirse que no se debe aceptar sin problemas que el lenguaje se limita a describir acontecimientos observables sin percatarse de que el lenguaje es mucho más que mero lenguaje descriptivo. Wittgenstein, que había explicado la

\footnotetext{
${ }^{22}$ Si bien Hume está dispuesto a añadir dentro de las cuestiones de hecho "la prueba", que se diferencia de lo probable en la constancia (distingue entre demostración, prueba y probabilidad).

23 "All ideas, especially abstract ones, are naturally faint and obscure: the mind has but a slender hold of them: they are apt to be confounded with other resembling ideas; and when we have often employed any term, though without a distinct meaning, we are apt to imagine it has a determinate idea annexed to it. On the contrary, all impressions, that is, all sensations, either outward or inward, are strong and vivid: the limits between them are more exactly determined: nor is it easy to fall into any error or mistake with regard to them. When we entertain, therefore, any suspicion that a philosophical term is employed without any meaning or idea (as is but too frequent), we need but enquire, from what impression is that supposed idea derived? And if it be impossible to assign any, this will serve to confirm our suspicion", An enquiry concerning human understanding, sección 2
} 
relación descriptiva entre los hechos atómicos y las proposiciones atómicas desde la isomorfía, se percató de que no podemos aceptar sin más el uso figurativo del lenguaje, ya que, por un lado el lenguaje es más que el lenguaje descriptivo, y por otro no tenemos porqué creer en la realidad de unos hechos atómicos de los que, por supuesto, no podemos hablar en tanto que los límites del lenguaje son los límites de nuestro mundo. Esta consideración llevó a Wittgenstein a la teoría de los usos del lenguaje según la cual el mundo está constituido por innumerables clases de expresiones e innumerables formas de usarlas.

Deben aceptarse sin miedo enunciados que van más allá de la mera percepción. Y además es difícil no hacerlo, incluso un empirista como Locke parece ya ir mucho más allá de lo que se ve y se toca cuando habla de cualidades reales, como el movimiento y el número, por ser objetivas. Considerar que lo objetivo es fundamento de lo subjetivo (las cualidades reales producen ideas simples -idea de rojo, frialdad...- que dan lugar a ideas complejas -percepción, duda...- e incluso a ideas abstractas -sin correlato empírico-) es ir más allá de la experiencia. Además resulta que, como sabrá hacer ver Husserl, seguramente deba decirse lo contrario y lo objetivo esté fundado en lo subjetivo, pues lo objetivo aparece como medida de la vivencia. El error es obviamente, confundir lo objetivo con lo real.

Por otro lado si ir más allá de los sentidos significa imaginar, entonces se pierde la posibilidad de la demostración (conocimiento científico) y pareciera que nos quedamos ante el escepticismo como única opción. Para salvar el escepticismo Kant había intentado responder a la pregunta por cómo pueden ser verdad los enunciados que expresan juicios sintéticos dando entrada a los juicios sintéticos a priori, necesarios y universales a la vez que amplían conocimiento.

Según Kant, el escepticismo de Hume comparece por no haber sabido distinguir este tipo de juicios. Este tipo de juicios permitiría por ejemplo que fueran aceptables enunciados como «todo suceso tiene una causa», que se distingue del juicio analítico «todo efecto tiene una causa». La explicación es que tales juicios se componen de intuiciones a priori. Pero esto significa que el entendimiento se convierte en el legislador de la naturaleza al imponer las leyes (a priori) a las que se somete la experiencia. Así, para Kant la síntesis será síntesis del entendimiento $^{24}$, y no síntesis lógica como en Aristóteles; cuyo Organon

${ }^{24}$ „denn sie ist ein Actus der Spontaneität der Vorstellungskraft, und da man diese zum Unterschiede von der Sinnlichkeit Verstand nennen muß, so ist alle Verbindung, wir 
ha sido sustituido por un Novum Organum (Bacon). Y aún llegará a decir Kant que todo uso del entendimiento está subordinado al papel fundamental de la unidad sintética de la apercepción: el Ich denke que acompaña a todas las representaciones de una manera ontológica. Pero en realidad lo que está en el origen de la aplicación de las categorías del entendimiento al caos de sensaciones espaciotemporales es la espontaneidad del esquematismo trascendental. La imaginación trascendental, el abismo ante el que Kant retrocederá, siendo Heidegger quien recuperará el análisis sobre la imaginación trascendental en la que reconocerá el carácter fundante de la espontaneidad del tiempo. Este retroceso kantiano conduce al idealismo hegeliano, la filosofía heideggeriana buscará dar el paso atrás para evitar el paso hacia el idealismo absoluto.

\section{Séptimo paso. El paso atrás hacia el ámbito presencial}

El idealismo es la filosofía que retrocede ante el poder de la imaginación. ¿Quién ha dicho que debamos aceptar sin más que ir más allá de la experiencia es obra de la imaginación necesariamente? El pensamiento que no necesita de la experiencia es el pensamiento puramente lógico ¿y si lo que es obra de la imaginación es la consideración de que existe auténtico conocimiento fuera de la lógica? Y

¿Quién ha dicho que al conocer conocemos la realidad y no el conocimiento mismo? Cuando digo de la pared que es verde ¿qué debo aprender? ¿Debo aprender que de la pared puede decirse que es verde, situando de esta manera el fundamento en el sujeto sobre el que se predica que es verde? ¿O debo aprender que puedo decir de la pared que es verde, porque yo (el sujeto que enuncia) veo verde? Ya vimos el camino que seguía cuando la respuesta era la primera alternativa. Ahora nos centraremos en la segunda alternativa tomando como representante a Hegel.

mögen uns ihrer bewußt werden oder nicht, es mag eine Verbindung des Mannigfaltigen der Anschauung oder mancherlei Begriffe, und an der ersteren der sinnlichen oder nichtsinnlichen Anschauung sein, eine Verstandeshandlung, die wir mit der allgemeinen Benennung Synthesis belegen würden, um dadurch zugleich bemerklich zu machen, da $\beta$ wir uns nichts als im Object verbunden vorstellen können, ohne es vorher selbst verbunden zu haben, und unter allen Vorstellungen die Verbindung die einzige ist, die nicht durch Objecte gegeben, sondern nur vom Subjecte selbst verrichtet werden kann, weil sie ein Actus seiner Selbstthätigkeit ist“, KRV, § 15, B130 
El pensamiento versa sobre sí, y sólo se somete a un aley: el rechazo de la contradicción. Por un lado pareciera que todo pensamiento tiene su opuesto, otro pensamiento que se le opone; pero al mismo tiempo parece que si decimos de algo que es verdad, entonces es porque no podemos decir lo opuesto. Así, si decimos que es verdad que la emisión de gases de los coches contamina, parece que no podemos afirmar su opuesto. La verdad, de este modo, implica la no existencia del opuesto (y por eso está llamado a perecer ${ }^{25}$ ). Por ello Aristóteles tiene que afirmar la actualidad de la sustancia, porque si yo digo de un sujeto que es $\mathrm{P}$, y esta proposición es verdadera, entonces, no puedo decir lo contrario y que siga siendo verdadero.

Desde este punto de vista se entiende que se considere que la no-contradicción implica una identidad. Habríamos encontrado la verdad, el sujeto verdadero.

Hegel considera, efectivamente, que todo pensamiento sobre algo tiene su opuesto, pero su dialéctica especulativa permite la superación ${ }^{26}$ hasta la consecución de la reunión de toda la verdad, y de este modo, el ya no de la oposición en la identidad de la sustancia que se ha transformado en sujeto ${ }^{27}$. La verdad sería así, aquel pensamiento que

25 „Das Zufällige, als die unmittelbare Wirklichkeit, ist zugleich die Möglichkeit eines Anderen, jedoch nicht mehr bloß jene abstrakte Möglichkeit, die wir zuerst hatten, sondern die Möglichkeit als seiend, und so ist dieselbe Bedingung. Wenn wir von der Bedingung einer Sache sprechen, so liegt darin zweierlei: einmal nämlich ein Dasein, eine Existenz, überhaupt ein Unmittelbares, und zweitens die Bestimmung dieses Unmittelbaren, aufgehoben zu werden und zur Verwirklichung eines Anderen zu dienen (...) als bloße Möglichkeit, ebenso bestimmt ist, aufgehoben $\mathrm{zu}$ werden. Als aufgehobene Möglichkeit ist sie das Hervorgehen einer neuen Wirklichkeit, welche die erste unmittelbare Wirklichkeit zu ihrer Voraussetzung hatte (...)In der Tat enthält aber solche unmittelbare Wirklichkeit den Keim zu etwas ganz anderem in sich. Dieses Andere ist zunächst nur ein Mögliches, welche Form sich dann aber aufhebt und in Wirklichkeit übersetzt (...) der Prozeß der Wirklichkeit“ Hegel, Enzyklopädie der philosophischen Wissenschaften im Grundrisse, $\$ 146$

26 "Seit der Epoche des spekulativen Idealismus bleibt es dem Denken untersagt, die Einheit der Identität als das bloße Einerlei vorzustellen und von der inr der Einheit waltenden Vermittelung abzusehen" Ga 11 p 34

${ }^{27}$ Ya el propio Platón, cometiendo un parricidio, había afirmado la existencia de la oposición en el sofista, aquel Platón viejo que había afirmado la existencia de la diferencia, del no-ser, cometiendo un parricidio que se sustentaba en el descubrimiento de que el no-ser no nombraba lo contrario al ser, sino lo diferente. El ser y el no-ser pueden pensarse como contrarios al modo como lo grande y lo pequeño son contrarios, pero el viejo Platón había visto que lo no-grande es mucho más que lo pequeño, es también lo igual, por ejemplo, y, de este modo, pudo afirmar la existencia del no-ser y la 
no es posible ser contradicho. La relación de identidad en este segundo caso tiene que ser lograda dialécticamente según la Aufhebung hegeliana hasta lograr la identidad del pensar consigo mismo.

Frente a esta verdad absoluta, meta del conocimiento, el análisis de la imaginación por parte de Heidegger en el esquematismo trascendental kantiano reconduce sin embargo la pregunta por la verdad hacia un camino bien diferente del tomado por el idealismo alemán en la medida en que el fundamento de la presencia no remite ni al sujeto presenciado ni al sujeto que presencia, sino hacia lo que podríamos denominar como ámbito presencial: el fundamento de la presencia no se encuentra en lo no-presente (clásico), ni en el presenciar (moderno), sino en lo presencial. Si algo no presente puede llegar a ser presenciado es porque ese algo es presenciable; siendo el fundamento de dicha presencialidad eso que hemos denominado el ámbito presencial, al que Heidegger denomina, en un primer momento, como mundo (que Heidegger piensa como desarrollo de la idea de tiempo que funciona en el esquematismo trascendental kantiano). No se debe confundir el mundo, tal y como lo concibe Heidegger, con la realidad extramental de lo no-presente. El mundo no es presenciable como la cosa no-presente, sino presencial. No es un conjunto de objetos en sí independientes de la experiencia, sino el carácter esencial de las cosas que las hace presenciables. O dicho de otra manera, si las cosas son presenciables es porque pertenecen al mundo (lo presencial). Que las cosas pertenecen al mundo significa que se encuentran insertas en una red remisional en las que cobran sentido. El sentido, de este modo se nos anticipa, la funcionalidad de las cosas, su ser-para nos viene dado por su pertenencia al mundo. También en la filosofía del segundo Heidegger se mantendrá ocupando un papel decisivo el pensamiento del ámbito presencial, si bien ya no lo denominará mundo (demasiado subjetivo, al fin y al cabo un existenciario), sino que hablará de Lichtung.

Este ámbito presencial no es una idea sólo presente en la filosofía heideggeriana. La encontramos por ejemplo ocupando un papel principal en la obra de Gadamer, donde se habla de tradición acontecida, o en la de Foucault, donde se habla de prácticas discursivas. La entrada a escena de dicho ámbito presencial hace que la pregunta por la verdad remita hacia ese contexto dentro del cual llega a aparecer algo como objeto de conocimiento. Hacia el horizonte de lo presente, el horizonte de

diferencia. Cfr., "¿acaso cuando hablamos de algo no-grande, te parece que con esta expresión designamos más a lo pequeño que a lo igual?” Sofista, 257b 
comprensión. Así, un enunciado del tipo «el oxígeno es un elemento atómico de número 8 y símbolo $\mathrm{O}$ » es un modo de hacerse presente que remite al contexto científico de la química actual. En un sentido similar Laudan hablará de tradiciones de investigación que cumplen las funciones de señalar qué supuestos no están sujetos a discusión, qué partes de una teoría deben ser modificadas, qué reglas deben establecerse y qué problemas conceptuales plantea una determinada teoría; esto es, especifica una ontología general y un método general de resolver los problemas dentro de un dominio determinado: "una tradición de investigación especifica una ontología general y un método general de resolver los problemas dentro de un dominio determinado" ${ }^{28}$.

Pero de este modo el horizonte dentro del cual comparece lo presente tiene una historia. Frente al sometimiento a esta historia, Nietzsche proclama el ejercicio creativo de la voluntad de poder como arte como mecanismo para sobre-ponernos a la historia. Claro que de este modo parece indicar el propio mecanismo por el cual se mantiene y perdura la propia transformación histórica de la verdad. Y, siendo así, el papel del hombre viene a ser más bien el de "pastor" que escucha su destino. Es el tema de la Cuadratura heideggeriana: ¿cómo acontece esa verdad hacia la que nos proyectamos, esa posibilidad que perseguimos?

Claro que esto que decimos va en contra de la estabilidad deseada por un espíritu positivista y conservador. ¿Habría que decir quizás que en pos de esta estabilidad, es mejor pensar de otra manera, y dejar de hablar de fundamentos y esencias? Tal cosa es lo que predicaba Compte para superar el estadio de lucha y sucesión histórica en el que nos instalaba la filosofía. Pero la ingenuidad del positivismo es considerar que es posible prescindir sin más del fundamento y la historia. Del ser no podemos prescindir, lo más que podemos hacer es olvidarlo. Y olvidarlo es la forma más cumplida de someterse al dictado de una época (de caer en el estado interpretativo del se impersonal). Si en última instancia toda pregunta por la verdad se levanta sobre la base histórica que la hace posible, lo que debe hacerse es reiterar la pregunta por el ser en busca delesclarecimiento del ámbito presencial donde se levanta la verdad de los pueblos. Tal fue la convicción heideggeriana cuyo grito emblema fue en el inicio die Frage nach dem Sinn vom Sein soll gestellt werden $^{29}$.

${ }^{28}$ Laudan, Progress and its problems, University of California Press, Berkeley, 1977, p 84

${ }^{29} \mathrm{GA} 2, \S 2$ 
El Dr. Alejandro Rojas Jiménez es investigador de la Alexander von Humboldt Stiftung en la Ludwig-Maximilians-Universität München y en la Bayerische Akademie der Wissenschaften (Alemania). También es colaborador honorario de la Universidad de Málaga, donde además es miembro delgrupo de investigación sobre el idealismo alemán: Schelling (HUM-172) de la Junta de Andalucía.

Algunas de sus últimas publicaciones son:

La Cuadratura. La última palabra ontológica de Heidegger, Universidad de Málaga, Málaga, 2009

Nota sobre la mismidad en la filosofía griega, en Contrastes XIII (2008)

$312-321$

Lebenswelt und Wissenschaft, en TABVLA RASA. Jenenser Zeitschrift für Kritisches Denken, Verlag Jena, in der Ausgabe 34 (IV/2008),

La libertad en la filosofía de la Cuadratura de Heidegger, en Thémata, 41(2009)

Informe bibliográfico sobre Heidegger, en Contrastes. Revista internacional de filosofía 14 (2009) 305-322

El paso en falso con respecto al cual no retrocede Heidegger, en Anuario filosófico, XLII/3 (2009), pp 627-647

Operaciones, y además hábitos, en González Ginocchio, David (ed), Metafísica y libertad, cuadernos de Anuario Filosófico serie universitaria, 214 (2009) pp 125-139

Lectura política del Der Spruch des Anaximander de Heidegger, en Intuslegere Filosofia, $\mathrm{n}^{\circ}$ 9, vol 3 (2009) 119-134 (Chile)

Este trabajo, Esbozo histórico acerca de la pregunta por la verdad, reproduce la ponencia de Alejandro Rojas del 6 de abril de 2010, en el centro cultural Vicente Aleixandre, Alhaurín de la Torre (Málaga), y que supuso el acto final con el que se cerró el ciclo de seminarios en torno a la verdad organizados por Claridades. Revista de filosofía en colaboración con el área de filosofía del departamento de filosofía de la Universidad de Málaga. 